Revista de Economia Política, vol. 31, $n^{\circ} 5$ (125), pp. 751-770, Edição especial/2011

\title{
Integração financeira, poupança externa e convergência de renda: teoria e evidência
}

ADERBAL OLIVEIRA DAMASCENO*

Financial integration, foreign savings and income convergence: theory and evidence. The conventional argument favoring capital controls elimination is based on the predictions from the neoclassical model: free international capital mobility would allow capital flows from country where capital is abundant to countries where capital is scarce and the outcome in a global perspective is efficient allocation of savings and income convergence. Within this perspective, financial integration would be particularly beneficial for developing countries resulting in external savings import, temporary increase in per-capita GDP growth rate and a permanent increase in the per-capita GDP level. Using data for a sample of 105 countries from 1980 to 2004 the evidences show that capitals flows from developing to developed countries and that international financial integration and external savings do not increase the conditional convergence rate.

Keywords: international financial integration; international capital flows; conditional convergence.

JEL Classification: F33; F36; F43.

\section{INTRODUÇÃO}

Com base no modelo neoclássico, a livre mobilidade de capitais permitiria o fluxo de capitais de países onde o capital é relativamente abundante para países onde o capital é relativamente escasso, o que resultaria, no âmbito global, em alocação eficiente da poupança e convergência de renda. Nessa perspectiva, a integração financeira internacional seria particularmente benéfica para os países em de-

\footnotetext{
* Professor do Instituto de Economia da Universidade Federal de Uberlândia. E-mail: damasceno@ ie.ufu.br. Submetido: 7/jul./2009; Aprovado: 29/mar./2010.
} 
senvolvimento, pois, supõe-se, nesses países o capital é escasso e o retorno marginal do capital é alto, de modo que a integração resultaria em importação de poupança externa, aumento temporário na taxa de crescimento do estoque de capital e do PIB per-capita e aumento permanente no nível do PIB per-capita.

$\mathrm{O}$ atual estágio da literatura no mainstream evidencia a ausência de consenso teórico e empírico acerca de benefícios da integração financeira internacional e dos fluxos internacionais de capitais para o crescimento econômico. Contudo, há um conjunto de lacunas na literatura econométrica, notadamente, a ausência de investigação sistemática acerca das relações entre integração financeira internacional e convergência condicional. Essa lacuna é relevante, pois o argumento clássico em defesa da eliminação de controles de capitais tem por base as previsões do modelo neoclássico, segundo o qual o efeito da integração sobre o crescimento do PIB per-capita é temporário, resultando em um aumento na taxa de convergência condicional.

O objetivo do artigo é realizar uma investigação econométrica acerca das relações entre integração financeira internacional, fluxo líquido de capitais e convergência condicional. Utilizando uma amostra de 105 países durante o período 1980-2004, serão estimadas equações de crescimento especificadas na forma de um modelo dinâmico de dados em painel. Os resultados sugerem que os capitais fluem dos países em desenvolvimento para os países desenvolvidos e que a integração financeira internacional e a importação líquida de capitais não aumentam a taxa de convergência condicional. Essas evidências não corroboram as previsões do modelo neoclássico e são, portanto, uma crítica contundente à tese de crescimento com poupança externa.

O trabalho está organizado em quatro seções, além desta introdução e das considerações finais. Na segunda seção faz-se uma revisão crítica da literatura teórica e empírica no âmbito do mainstream acerca das relações entre integração financeira internacional, fluxos internacionais de capitais e crescimento econômico. $\mathrm{Na}$ terceira seção faz-se uma investigação acerca do padrão de integração financeira internacional e dos fluxos internacionais de capitais em âmbito global. Na quarta seção apresentam-se os procedimentos metodológicos utilizados para a realização da investigação econométrica. Por fim, na quinta seção, são apresentados e discutidos os resultados das estimações econométricas.

\section{INTEGRAÇÃO FINANCEIRA INTERNACIONAL E CRESCIMENTO ECONÔMICO}

\section{A literatura teórica}

O argumento clássico em defesa da eliminação de controles sobre os fluxos de capitais tem por base as previsões do modelo neoclássico (Solow-Swan/Ramsey-Cass-Koopmans): a livre mobilidade internacional de capitais permitiria o fluxo de capitais de países onde o capital é relativamente abundante e o retorno marginal 
do capital é baixo para países onde o capital é relativamente escasso e o retorno marginal do capital é alto, resultando em uma alocação eficiente da poupança e convergência de renda em âmbito global. Nesse modelo teórico a integração financeira internacional tem implicações para o padrão dos fluxos internacionais de capitais, acumulação de capital e crescimento do PIB per-capita (Gourinchas e Jeanne, 2006; Henry, 2007; Acemoglu, 2009):

i) os países desenvolvidos, onde o capital é relativamente abundante e o retorno marginal do capital é baixo, deveriam exportar capital. Os países em desenvolvimento, onde o capital é relativamente escasso e o retorno marginal do capital é alto, deveriam importar capital. Entre os países em desenvolvimento, o capital deveria fluir para aqueles com maior crescimento da produtividade total dos fatores;

ii) nos países desenvolvidos, a integração resultaria em alto retorno para a poupança doméstica, diminuição temporária na taxa de crescimento do estoque de capital e do PIB per-capita e aumento na taxa de crescimento do PNB. Nos países em desenvolvimento, a integração resultaria em aumento temporário na taxa de crescimento do estoque de capital e do PIB per-capita e aumento permanente no nível do PIB per-capita.

No modelo neoclássico, a integração financeira internacional não altera a taxa de crescimento da produtividade total dos fatores e, portanto, a taxa de crescimento do PIB per-capita no estado estacionário (Henry, 2007). O canal por meio do qual a integração financeira internacional estimula o crescimento do PIB per-capita nas economias domésticas é a acumulação de capital e, desse modo, a integração aumenta a taxa de convergência (condicional) em direção ao estado estacionário, que seria o mesmo sob autarquia (Gourinchas e Jeanne, 2006).

Ademais, assume-se que o mercado financeiro internacional é eficiente e que não existem distorções nas economias domésticas (Henry, 2007). A assunção da existência de distorções no mercado financeiro internacional (assimetria de informação e problemas de enforcement de contratos) e nas economias domésticas (baixo nível de desenvolvimento institucional, de desenvolvimento financeiro, de abertura comercial e de estabilidade macroeconômica) permite um questionamento aos fundamentos teóricos deste modelo.

Eichengreen et al. (1998) admitem que a existência de assimetria de informação, problemas de enforcement de contratos e distorções nas economias domésticas enfraquecem os pressupostos teóricos subjacentes ao modelo neoclássico, mas sugerem a possibilidade de que a integração ao mercado financeiro internacional estimula o crescimento do PIB per-capita apenas em países com um alto nível de desenvolvimento institucional, de desenvolvimento financeiro, de abertura comercial e de estabilidade macroeconômica.

Rodrik (1998), Bhagwati (1998) e Stiglitz (2000) afirmam que está subjacente ao modelo neoclássico a analogia entre o livre comércio de ativos financeiro e o livre comércio de bens. Segundo os autores, o comércio de ativos financeiros, por conta da existência de assimetria de informação, é essencialmente distinto do comércio de bens e serviços, de modo que as previsões do modelo neoclássico são 
falsas. Ademais, argumenta Stiglitz (2000, p. 1080), “[...] there are even reasons to expect that capital market liberalization can have negative effects on growth".

Rodrik e Subramanian $(2009$, p. 124) afirmam que os países em desenvolvimento sofrem de múltiplas distorções e questionam o argumento, defendido por Eichengreen (2007), a respeito da possibilidade de que a integração financeira internacional estimule o crescimento econômico em países onde existam condições iniciais adequadas: "Finally, we have doubts with respect to the theoretical consistency and practical feasibility of the regulatory and other reforms needed to support financial globalization in the kinds of environments faced by developing nations".

Para Bresser-Pereira e Gala (2007) e Rodrik e Subramanian (2009), o principal constrangimento ao crescimento econômico nos países em desenvolvimento não é ausência de poupança, mas de oportunidades de investimento. Com base em arcabouços teóricos distintos, argumentam que a entrada de capitais (poupança externa) resulta na apreciação da taxa de câmbio real, a qual exacerba esse constrangimento, estimula o crescimento do consumo, a substituição da poupança interna pela externa e pode, inclusive, desestimular o investimento e o crescimento econômico ${ }^{1}$.

\section{A literatura empírica}

Desde o final da década de 1990 é crescente o esforço de pesquisa com o objetivo de verificar empiricamente a existência de benefícios advindos da integração financeira internacional. No geral, os trabalhos estimam equações de crescimento incluindo entre as variáveis explanatórias algum índice (de jure ou de facto) de integração financeira internacional.

Dentre os trabalhos que não encontram evidências de que a integração financeira internacional e os fluxos internacionais de capitais estimulam o crescimento econômico de longo prazo destacam-se Grilli e Milesi-Ferretti (1995), Rodrik (1998), Kraay (1998), Prasad et al. (2007) e, especialmente, Carkovic e Levine (2005) e Edison et al. (2002). Em conjunto, esses trabalhos não encontram evidências de que a integração financeira internacional e os fluxos internacionais de capitais estimulam o crescimento de longo prazo do PIB per-capita, mesmo em países com alto nível de desenvolvimento econômico, de desenvolvimento institucional, de desenvolvimento financeiro, de abertura comercial, de estabilidade macroeconômica e do estoque de capital humano.

Areta et al. (2001) encontram evidências frágeis de que a integração financeira internacional estimula o crescimento econômico de longo prazo do PIB per-capita, mesmo para países com alto nível de PIB per-capita, de desenvolvimento institucional, de desenvolvimento financeiro e de abertura comercial. Edison et al.

\footnotetext{
${ }^{1}$ Trabalhos realizados no âmbito do Fundo Monetário Internacional esboçam uma abordagem distinta do modelo neoclássico, ao sugerirem a existência de uma relação entre integração financeira internacional e crescimento de longo prazo do PIB per-capita (Prasad et al. 2003; Dell'Ariccia et al. 2008; Kose et al. 2009). Neste artigo não examinaremos o status teórico dessa abordagem e as implicações empíricas.
} 
(2004) não encontram evidências de que a integração financeira internacional e, mais especificamente, a liberalização do mercado de capitais, estimula o crescimento econômico, exceto para um pequeno grupo de países de renda média.

Dentre os trabalhos que encontram evidências de que a integração financeira internacional estimula o crescimento econômico, destacam-se Quinn (1997) e Bekaert et al. (2005). Os resultados apresentados em Quinn (1997) seriam, segundo o autor, a primeira demonstração sistemática de que a integração financeira internacional estimula o crescimento econômico de longo prazo. Bekaert et al. (2005) encontram evidências de que a liberalização do mercado de capitais estimula o crescimento econômico de longo prazo e de que esse estímulo é mais pronunciado em países com alto nível de desenvolvimento institucional.

Essa pequena síntese da literatura econométrica sugere a inexistência de evidências sistemáticas de que a integração financeira internacional e os fluxos internacionais de capitais estimulam crescimento econômico de longo prazo. Em uma crítica a essa literatura, Henry (2007) argumenta que a estimação de equações de crescimento utilizado índices de integração financeira internacional como variável explanatória testa a relação entre integração financeira internacional e crescimento econômico de longo prazo do PIB per-capita e, portanto, não testa o modelo neoclássico, segundo o qual a integração financeira internacional aumenta a taxa de convergência condicional.

$\mathrm{Na}$ literatura econométrica, dois trabalhos testam a relação entre integração financeira internacional e convergência condicional. McKenzie (2001), com dados para uma amostra de 112 países no período 1960-1989, encontra evidências frágeis de que a taxa de convergência é menor em países que impõem controles de capitais. Klein (2005, p. 24), com dados para uma amostra de 71 países no período 1976-1995, conclui que a hipótese de que “[...] an open economy experiences more rapid conditional convergence than a closed economy, is not consistent with the empirical results".

Gourinchas e Jeanne (2006, p. 716) adotam uma abordagem empírica distinta, calibram um modelo neoclássico de crescimento na versão Ramsey-Cass-Koopmans para avaliar os benefícios da integração financeira internacional para um típico país em desenvolvimento advindos do aumento da convergência condicional. As conclusões são claras: "[...] we find that the gains from international financial integration may be relatively small even for countries that stand to receive a lot of capital inflows".

A crítica de Henry (2007) ressalta para um aspecto relevante e sobre o qual a literatura econométrica não dá a devida atenção, dada a ausência de investigação econométrica sistemática acerca da relação entre integração financeira internacional e convergência condicional. Esse trabalho contribui para a literatura por meio de uma análise empírica sobre o padrão dos fluxos internacionais de capitais e de uma investigação econométrica acerca das relações entre integração financeira internacional, fluxos internacionais de capitais e convergência condicional. 


\section{INTEGRAÇÃO FINANCEIRA INTERNACIONAL E FLUXOS INTERNACIONAIS DE CAPITAIS}

Três conjuntos de transformações estão subjacentes ao crescente grau de mobilidade dos fluxos internacionais de capitais desde o colapso do arcabouço institucional erigido em Bretton Woods: i) decisões de política econômica relativas à eliminação dos controles legais sobre os fluxos internacionais de capitais; ii) avanços tecnológicos, especialmente nos setores de informática e de tecnologias de processamento de informações; e iii) proliferação das inovações financeiras, a securitização das dívidas e a institucionalização das poupanças.

Os Gráficos 1 e 2 apresentam o comportamento dos índices de integração financeira internacional de jure CIIFI e de facto LMFIFI ao longo do período 1970-2004 para uma amostra de 22 países desenvolvidos e 83 países em desenvolvimento. Há inequívoca tendência em direção a um maior nível de integração financeira internacional em âmbito global. Ademais, relativamente aos países desenvolvidos, a integração dos países em desenvolvimento ao mercado financeiro internacional é tardia e em menor intensidade ${ }^{2}$.

Gráfico 1: Integração Financeira Internacional de Jure (CIIFI)

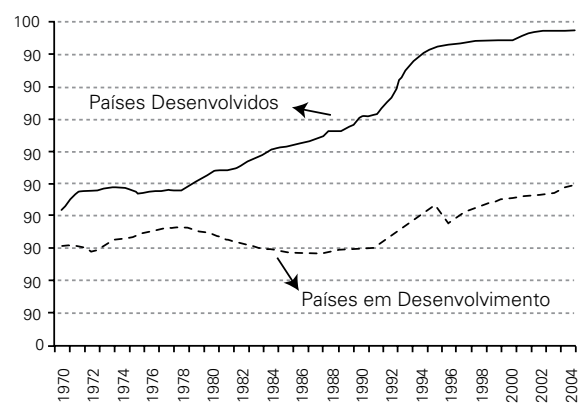

Gráfico 2: Integração Financeira Internacional de Jure (LMFIFI)

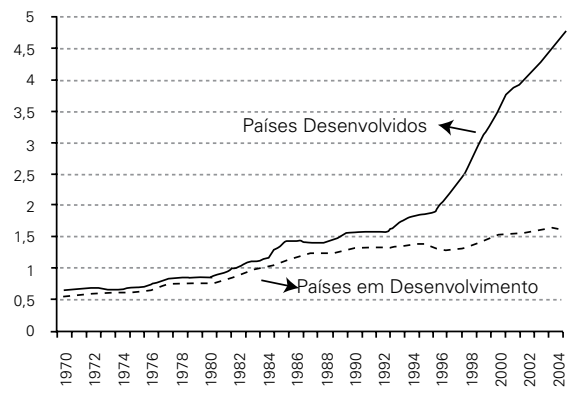

O Gráfico 3 mostra o comportamento do Estoque Total de Obrigações para uma amostra de 22 países desenvolvidos e 83 países em desenvolvimento ao longo do período 1970-2004. O Estoque Total de Obrigações dos grupos de países desenvolvidos e em desenvolvimento correspondia, respectivamente, a US\$ 446 bilhões e US\$ 117 bilhões em 1970 e US\$ 46,666 trilhões e US\$ 6,271 trilhões em 2004. Em 2004, o Estoque Total de Obrigações dos grupos de países desenvolvidos

\footnotetext{
${ }^{2} \mathrm{O}$ gráfico para o índice de integração financeira internacional de jure SEIFI mostra o mesmo padrão do Gráfico 1 e o gráfico para o índice de integração financeira de facto WDIFI mostra o mesmo padrão do Gráfico 2, por isso não foram reportados aqui. As definições detalhadas dos índices de integração financeira internacional de jure CIIFI e SEIFI e de facto LMFIFI e WDIIFI são apresentadas na quarta seção, que trata dos procedimentos metodológicos adotados para a realização dos cálculos econométricos, apresentados e discutidos na quinta seção.
} 
e em desenvolvimento correspondia, respectivamente, a 106 vezes e 53 vezes o valor em 1970 .

O Gráfico 4 mostra o comportamento do Fluxo Total de Capitais para uma amostra de 22 países desenvolvidos e 83 países em desenvolvimento ao longo do período 1980-2004. O Fluxo Total de Capitais direcionado aos grupos de países desenvolvidos e em desenvolvimento correspondia, respectivamente, a US\$ 426 bilhões e US\$ 60 bilhões em 1980 e US\$ 4,3 trilhões e US\$ 0,408 trilhões em 2004. Em 2004, o Fluxo Total de Capitais direcionado aos grupos de países desenvolvidos e em desenvolvimento correspondia, respectivamente, 10 vezes e 6 vezes o valor em 1980 .

Os dados explicitam o caráter assimétrico do sistema financeiro internacional: i) o Estoque Total de Obrigações do grupo de países em desenvolvimento é, respectivamente, 26\% em 1970 e 13\% em 2004, do Estoque Total de Obrigações dos países desenvolvidos; ii) o Fluxo Total de Capitais direcionado aos países em desenvolvimento é, respectivamente, 14\% em 1980 e 9\% em 2004, do Fluxo Total de Capitais direcionado aos países desenvolvidos. A participação dos países em desenvolvimento no mercado financeiro internacional é marginal e essa assimetria foi exacerbada com a formação de um mercado financeiro internacional integrado.

Gráfico 3:

Estoque total de obrigações (US\$ bilhões)

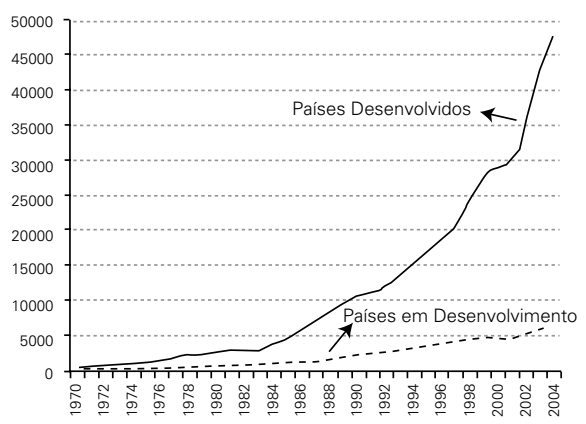

Gráfico 4:

Estoque total de capitais (US\$ bilhões)

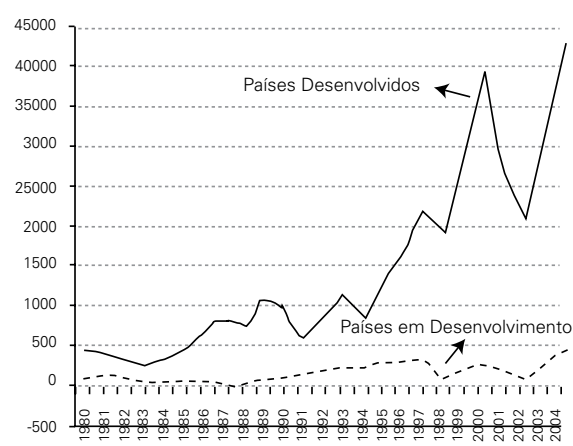

Os países desenvolvidos, onde o capital seria abundante e o retorno marginal do capital baixo, deveriam ser exportadores líquidos de capitais. Os países em desenvolvimento, onde o capital seria escasso e o retorno marginal do capital alto, deveriam ser importadores líquidos de capitais. O comportamento da Conta-Corrente para esses dois grupos de países permite ilustrar a incoerência entre o padrão efetivo dos fluxos internacionais de capitais e as previsões do modelo neoclássico. Os Gráficos 5 e 6 apresentam o comportamento do saldo em Conta-Corrente para 22 países desenvolvidos e 83 países em desenvolvimento ao longo de 1980-2004.

O grupo de países desenvolvidos apresenta déficits em Conta-Corrente ao longo de praticamente todo o período, especialmente a partir de 1998. O saldo anual médio em Conta-Corrente para esse grupo de países é US\$ -31,480 bilhões 
no período 1980-1997, US\$ -332,991 bilhões no período 1998-2007 e US\$ -139,163 bilhões no período 1980-2007. O saldo em Conta-Corrente acumulado para esse grupo de países é US\$ -566,647 bilhões no período 1980-1997, US\$ -3.329 bilhões no período 1998-2007 e US\$ - 3.896,566 bilhões no período 19802007. Ou seja, o grupo de países desenvolvidos é importador líquido de capitais no período.

O grupo de países em desenvolvimento apresenta pequenos déficits em Conta-Corrente ao longo do período 1981-1998 e grandes superávits a partir de 1998. O saldo anual médio em Conta-Corrente para esse grupo de países é US\$ -44,354 bilhões no período 1980-1997, US\$251,850 bilhões no período 1998-2007 e US\$ 61,432 bilhões no período 1980-2007. O saldo acumulado em Conta-Corrente para esse grupo de países é US\$ $-798,388$ bilhões no período 1980-1997, US\$ 2.518,507 bilhões no período 1998-2007 e US\$ 1.720,119 bilhões no período 1980-2007. Ou seja, o grupo de países em desenvolvimento é exportador líquido de capitais no período.

Gráfico 5:

Saldo em transações correntes (US\$ bilhões)

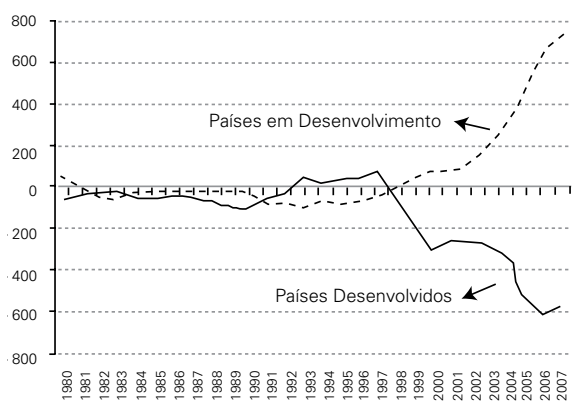

Gráfico 6:

Saldo em transações correntes (\% PIB)

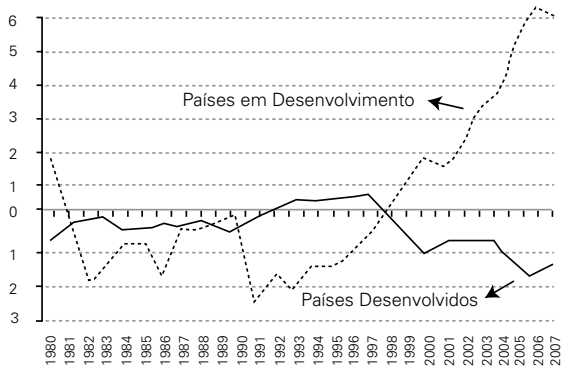

Deveria existir uma correlação negativa entre o nível inicial de renda per-capita e a entrada de capitais no período posterior: os capitais deveriam fluir para os países onde o nível inicial de renda per-capita é baixo, o nível inicial do estoque de capital per-capita é baixo e o retorno marginal do capital é alto. Em uma amostra de países desenvolvidos e em desenvolvimento, a correlação entre entrada de capitais per-capita e nível inicial de renda per-capita permite ilustrar a incoerência entre o padrão efetivo dos fluxos internacionais de capitais e as previsões do modelo neoclássico.

O Gráfico 7 mostra a associação entre o PIB real per-capita em 1980 e o Estoque Total de Obrigações per-capita (média anual para o período 1980-2004, com a linha de ajuste (OLS) e o intervalo de confiança de 95\%) - o coeficiente de correlação é 0,92. O Gráfico 8 mostra a associação entre o PIB real per-capita em 1980 e Fluxo Total de Capitais per-capita (média anual para o período 1980-2004, com a linha de ajuste (OLS) e o intervalo de confiança de $95 \%$ ) - o coeficiente de correlação é 0,84 . Dessa maneira, os países onde o nível do PIB real per-capita é 
maior em 1980 recebem uma quantidade maior de capitais no período posterior. Essa é uma evidência de que os capitais fluem para os países ricos.

Gráfico 7:

Estoque de obrigações per-capita

e PIB per-capita

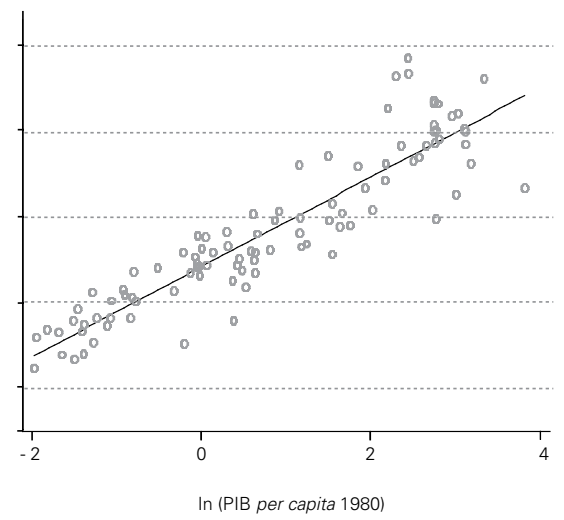

Gráfico 8:

Fluxo total de capitais per-capita

e PIB per-capita

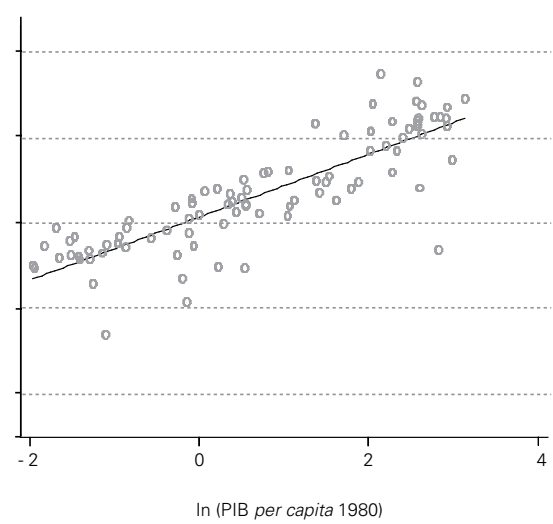

O comportamento do Estoque Total de Obrigações, Fluxo Total de Capitais, Conta-Corrente e a relação entre PIB per-capita e Fluxo Total de Capitais per-capita (Estoque Total de Obrigações per-capita) ilustram a incoerência entre as previsões do modelo neoclássico e o padrão efetivo dos fluxos internacionais de capitais. A ausência de fluxos de capitais em grandes quantidades dos países desenvolvidos para os países em desenvolvimento foi documentada por Lucas (1990) e considerada um puzzle. Os dados apresentados revelam uma incoerência ainda mais profunda: os capitais não fluem pouco dos países desenvolvidos para os países em desenvolvimento (Lucas Puzzle), eles fluem dos países em desenvolvimento para os países desenvolvidos.

Gourinchas e Jeanne (2008, p. 2), tendo por base o modelo neoclássico ( $R a$ msey-Cass-Koopmans) derivam uma relação entre os fluxos internacionais de capitais direcionados aos países em desenvolvimento e o crescimento da produtividade total dos fatores: "We show that a textbook model delivers a simple answer to this question: capital should flow into the developing countries whose TFP catches up relative to advanced economies, and should flow out of the countries whose TFP fall behind". Ou seja, os capitais deveriam fluir para os países em desenvolvimento com maior crescimento da produtividade total dos fatores.

Tendo como base uma amostra de 69 países em desenvolvimento, o Gráfico 9 mostra a relação entre fluxo líquido de capitais (saldo em Conta-Corrente, \% do PIB) e crescimento da produtividade total dos fatores (médias anuais para o período 1980-2000, com a linha de ajuste (OLS) e o intervalo de confiança de 95\%) - o coeficiente de correlação é 0,43 . Fica evidente que os países onde o crescimento da produtividade total dos fatores é maior recebem uma menor quantidade de capitais. Esse é, conforme Gourinchas e Jeanne (2008), o Allocation Puzzle. 
Gráfico 9: Estoque de capitais e produtividade

Fluxo Líquido de Capitais e Produtividade

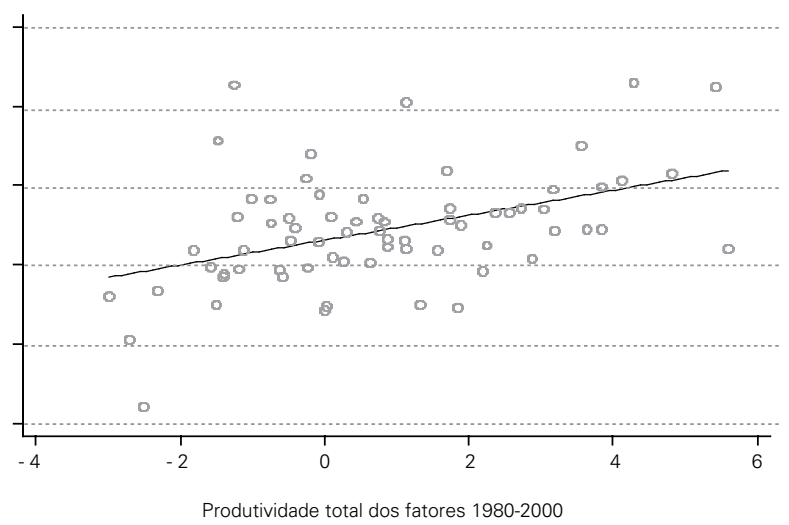

Os dados apresentados nessa seção evidenciam a incoerência entre o padrão efetivo dos fluxos internacionais de capitais e as previsões do modelo neoclássico: i) os capitais fluem, em termos líquidos, dos países em desenvolvimento para os países desenvolvidos; ii) os capitais fluem para os países em desenvolvimento com menor crescimento da produtividade total dos fatores.

\section{PROCEDIMENTOS METODOLÓGICOS}

A equação de crescimento está especificada na forma de um modelo dinâmico de dados em painel:

$$
\begin{aligned}
& y_{i, t}-y_{i, t-1}=(\alpha-1) y_{i, t-1}+x_{i, t}^{\prime} \beta+u_{i, t} \quad i=1,2, \ldots, N \text { e } t=2,3, \ldots, T \\
& u_{i, t}=\gamma_{t}+\eta_{i}+v_{i, t}
\end{aligned}
$$

Onde $y_{i, t}$ é o logaritmo natural do PIB per-capita para o país $i$ no período $t$, $y_{i, t-1}$ é o logaritmo natural do PIB per-capita para o país $i$ no período $t$-1, $x_{i, t}^{\prime}$ é um vetor-linha de possíveis determinantes do estado estacionário para o país $i$ no período $t$ e $u_{i, t}$ é o termo de erro. O termo $\gamma_{t}$ varia ao longo do tempo, é constante entre os países e capta choques comuns a todos os países e não controlados pelas variáveis em $x_{i, t}^{\prime}$. O termo $\eta_{i}$, país específico, varia entre os países, é constante ao longo do tempo e controla para determinantes do crescimento não incluídos em $x_{i, t}^{\prime}$. O termo $v_{i, t}$ varia entre os países e ao longo do tempo.

A variável dependente é a taxa de crescimento do PIB per-capita real $\left(v_{i, t}-y_{i, t-1}\right)$, calculada como a diferença do logaritmo natural do PIB per capta entre o primeiro e último ano de cada período de cinco anos e o PIB per-capita inicial $\left(y_{i, t-1}\right)$ é calculado como o logaritmo natural do PIB per-capita no ano inicial de cada período de cinco anos (US\$ constante de 2000, World Development Indicators, 2007). As variáveis de controle incluídas em $x_{i, t}^{\prime}$ são: 
i) Capital Humano - média dos anos de escolaridade da população com idade superior a 15 anos e é incluída na equação de crescimento como o nível no ano inicial de cada período de cinco anos (Barro e Lee, 2001).

ii) Abertura Comercial - soma de importações + exportações de bens e serviços como \% do PIB e é incluída na equação de crescimento como a média para cada período de cinco anos (World Development Indicators, 2007).

iii) Lei \& Ordem - proxy para a qualidade do ambiente institucional, incluída na equação de crescimento como a média para cada período de cinco anos (Political Service Risk Group, 2008).

iv) Inflação - variação anual percentual do índice de preço ao consumidor e é incluída na equação de crescimento como média para cada período de cinco anos (World Development Indicators, 2007).

v) Governo - gastos do governo em consumo como \% do PIB e é incluída na equação de crescimento como a média para cada período de cinco anos (World Development Indicators, 2007).

vi) CIIFI - informa a existência ou inexistência de controles legais sobre os fluxos internacionais de capitais e a intensidade de enforcement desses controles. É um índice de integração financeira internacional de jure, a escala é 0-100 e um maior valor indica maior grau de integração (Chinn e Ito, 2007).

vii) SEIFI - informa a existência ou inexistência de controles sobre os fluxos internacionais de capitais e a intensidade de enformement desses controles. É um índice de integração financeira internacional de jure, a escala é 0-100 e um maior valor indica maior grau de integração (Edwards, 2005 e 2007).

viii) $L M F I F I$ - é a soma de estoque de ativos e obrigações relativo a Investimento Externo Direto, Investimento de Portfólio, Outros Investimentos e Derivativos Financeiros como proporção do PIB. É um índice de integração financeira internacional de facto e um maior valor indica maior grau de integração (Lane e Milesi-Ferretti, 2007).

ix) WDIIFI - é a soma do fluxo de entrada e saída de Investimento Externo Direto, Investimento de Portfólio e Outros Investimentos, como \% do PIB. É um índice de integração financeira internacional de facto e um maior valor indica maior grau de integração (World Development Indicators, 2007).

x) FLCTC - fluxo líquido de capitais, é o saldo em Transações Correntes do Balanço de Pagamentos, como \% do PIB (World Economic Outlook Database, 2008).

A amostra é composta de 105 países, 22 países desenvolvidos e 83 países em desenvolvimento. Países desenvolvidos: Austrália, Áustria, Bélgica, Canadá, Dinamarca, Finlândia, França, Alemanha, Grécia, Islândia, Irlanda, Itália, Japão, Holanda, Nova Zelândia, Noruega, Portugal, Espanha, Suécia, Suíça, Reino Unido, Estados Unidos. Países em desenvolvimento: Argentina, Brasil, Chile, China, Colômbia, Egito, Hong Kong, Índia, Indonésia, Israel, Jordânia, Coreia, Malásia, México, Paquistão, Peru, Filipinas, Arábia Saudita, Cingapura, África do Sul, Tailândia, Turquia, Venezuela, Argélia, Bahrein, Bangladesh, Benin, Bolívia, Botsuana, Burkina Faso, Camarões, Chade, Dem. Rep. do Congo, Rep. do Congo, Costa 
Rica, Costa do Marfim, República Dominicana, Equador, El Salvador, Etiópia, Fiji, Garbon, Gana, Guatemala, Guiné, Haiti, Honduras, Irã, Jamaica, Quênia, Kuwait, Líbano, Líbia, Madagáscar, Malawi, Mali, Malta, Maurícios, Marrocos, Birmânia, Nepal, Nicarágua, Níger, Nigéria, Omã, Panamá, Papua-Nova Guiné, Paraguai, Catar, Ruanda, Senegal, Sri Lanka, Sudão, Suazilândia, Síria República Árabe, Tanzânia, Togo, Trindade e Tobago, Tunísia, Uganda, Emirados Árabes Unidos, Uruguai e Zâmbia.

As informações utilizadas para a estimação das equações de crescimento são organizadas em cinco períodos de cinco anos não sobrepostos (1980-1984, 1985-1989, 1990-1994, 1995-1999, 2000-2004). O painel é desequilibrado, de modo que não temos todas as observações relativas a todas as variáveis em todos os períodos para todos os países. Para cada unidade de observação tem-se: i) a taxa de crescimento é calculada para cada período de cinco anos; ii) o logaritmo do nível inicial do PIB per capita e Capital Humano são calculados no início de cada período de cinco anos; iii) as variáveis Comércio, Lei \& Ordem, Inflação, Governo, CIIFI, SEIFI, LMFIFI, WDIIFI e FLCTC e as variáveis de interação são mensuradas como a média para cada período de cinco anos.

As equações de crescimento serão estimadas por meio dos estimadores Difference GMM e System GMM, desenvolvidos por Arellano e Bond (1991), Arellano e Bover (1995) e Blundell e Bond (1998). A consistência do estimador GMM depende da validade das condições de momento. Para tanto, serão considerados três testes de especificação sugeridos por Arellano e Bond (1991), Arellano e Bover (1995) e Blundell e Bond (1998): i) o Sargan Test e o Diff Sargan Test, que testam a validade das condições de momento; ii) e o Arellano-Bond Test, que testa a hipótese de inexistência de correlação serial de segunda ordem no termo de erro.

Três aspectos justificam a utilização dos estimadores Difference GMM e System GMM: i) explorar a natureza temporal da relação entre as variáveis explanatórias e a taxa de crescimento do PIB per-capita; ii) controlar para efeitos específicos a cada país não observáveis e choques específicos a cada período comuns a todos os países; iii) obter estimativas consistentes e assintoticamente eficientes dos parâmetros de interesse, mesmo ao assumir a possível endogeneidade de todas as variáveis explanatórias. As estimações serão realizadas por meio do software Stata 10, utilizando o comando xtabond2 desenvolvido por Roodman (2009).

\section{INTEGRAÇÃO FINANCEIRA INTERNACIONAL, FLUXO LÍQUIDO DE CAPITAIS E CONVERGÊNCIA CONDICIONAL: EVIDÊNCIA ECONOMÉTRICA}

\section{Estratégia empírica}

Em uma equação de crescimento, o coeficiente associado ao logaritmo do PIB per-capita no ano inicial de cada intervalo de tempo informa sobre a existência de convergência condicional, uma vez que a equação inclua variáveis explanatórias 
que controlam para os determinantes do estado estacionário. Se o coeficiente associado ao nível inicial do PIB per-capita for negativo e estatisticamente significativo, existe evidência de convergência condicional (Barro e Sala-i-Martin, 2004; Acemoglu, 2009).

Para testar a hipótese de que a integração financeira internacional aumenta a taxa de convergência condicional, far-se-á a taxa de convergência condicional contingente ao nível de integração financeira internacional. Para tanto, serão construídas variáveis de interação entre o logaritmo do nível inicial do PIB per-capita e os índices de integração financeira internacional e a medida de fluxo líquido de capitais (Aghion et al., 2005; McKenzie, 2001; Klein, 2005). As variáveis de interação são: PIB inicial x CIIFI, PIB inicial x SEIFI, PIB inicial x LMFIFI, PIB inicial x WDIIFI e PIB inicial x FLCTC.

Se o coeficiente associado à variável de interação entre o nível inicial do PIB per-capita e um índice de integração financeira internacional for negativo e estatisticamente significativo, existe evidência de que um aumento no nível de integração financeira internacional aumenta a taxa de convergência condicional. Se o coeficiente associado à variável de interação entre o nível inicial do PIB per-capita e a medida de fluxo líquido de capitais for positivo e estatisticamente significativo, existe evidência de que a importação líquida de capitais aumenta a taxa de convergência condicional.

Nas Tabelas 1 a 5, em Apêndice, as colunas (1) a (4) apresentam resultados para a amostra total de países e as colunas (5) a (8) para a amostra de países em desenvolvimento. Ademais, em cada tabela, nas colunas (1), (3), (5) e (7), as equações são estimadas por System GMM, e nas colunas (2), (4), (6) e (8) as equações são estimadas por Difference GMM. Por fim, em cada uma das tabelas, são apresentados os testes de especificação AR(2), Hansen Test e Diff. Hansen Test para cada equação estimada. Dentre as 40 equações estimadas, apenas o teste $A R(2)$, na coluna (1) das Tabelas 2, 3 e 4, rejeita a especificação da equação.

\section{Integração financeira internacional de jure e convergência condicional}

A Tabela 1 apresenta o resultado de regressões que testam a relação entre integração financeira internacional e convergência condicional utilizando o índice de jure CIIFI. Exceto na equação apresentada na coluna 5, em todas as demais equações estimadas o coeficiente associado à variável de interação PIB inicial x CIIFI é negativo, mas não é estatisticamente significativo em nenhuma das 8 equações estimadas.

A Tabela 2 apresenta o resultado de regressões que testam a relação entre integração financeira internacional e convergência condicional utilizando o índice de jure SEIFI. Nas equações apresentadas nas colunas (1), (3), (7) e (8) o coeficiente associado à variável de interação PIB inicial x SEIFI é negativo, mas não é estatisticamente significativo em nenhuma das 8 equações estimadas.

As evidências apresentadas para a amostra total de países e para a amostra de países em desenvolvimento, utilizando os índices de jure CIIFI e SEIFI, não corro- 
boram a hipótese de que a eliminação de controles sobre os fluxos internacionais de capitais aumenta a taxa de convergência condicional nas economias nacionais.

\section{Integração financeira internacional de facto e convergência condicional}

A Tabela 3 apresenta o resultado de regressões que testam a relação entre integração financeira internacional e convergência condicional utilizando o índice de facto LMFIFI. Apenas nas equações apresentadas nas colunas (3), (4) e (8) o coeficiente associado à variável de interação PIB inicial x LMFIFI é negativo, mas não é estatisticamente significativo em nenhuma das 8 equações estimadas.

A Tabela 4 apresenta o resultado de regressões que testam a relação entre integração financeira internacional e convergência condicional utilizando o índice de facto WDIIFI. Apenas na regressão apresentada na coluna (3) o coeficiente associado à variável de interação PIB inicial x WDIIFI é negativo e, nas quatro regressões apresentadas para a amostra de países em desenvolvimento, o coeficiente é positivo e estatisticamente significativo, sugerindo que um aumento na integração financeira internacional diminui a taxa de convergência condicional.

Portanto, as evidências apresentadas para a amostra total de países e para a amostra de países em desenvolvimento, utilizando os índices de facto LMFIFI e WDIIFI, não corroboram a hipótese de que a integração ao mercado financeiro internacional aumenta a taxa de convergência condicional nas economias nacionais.

\section{Fluxo líquido de capitais e convergência condicional}

A Tabela 5 apresenta o resultado de regressões que testam a relação entre fluxo líquido de capitais (FLCTC), como mensurado pelo saldo em Conta-Corrente, e convergência condicional. Como pode ser observado, o coeficiente associado à variável de interação PIB inicial x FLCTC é positivo nas regressões apresentadas nas colunas (1), (3), (5), (6), (7), (8), mas não é estatisticamente significativo em nenhuma das 8 equações apresentadas.

Dessa maneira, os resultados apresentados para a amostra total de países e para a amostra de países em desenvolvimento, não corroboram a hipótese de que a importação de poupança externa aumenta a taxa de convergência condicional. Essas são, portanto, evidências que questionam a tese de crescimento com poupança externa.

\section{CONSIDERAÇÕES FINAIS}

Este trabalho contribui para a literatura sobre integração financeira internacional por meio de uma análise empírica do padrão dos fluxos internacionais de capitais e da realização de uma investigação econométrica sistemática acerca das relações entre integração financeira internacional, fluxos internacionais de capitais e convergência condicional. Ademais, o trabalho apresenta avanços metodológicos 
relevantes em relação à literatura: i) utilizam-se os estimadores Difference GMM e System GMM; ii) utilizam-se uma amostra de países representativa da economia global e uma amostra de países em desenvolvimento; iii) os dados utilizados abrangem o período 1980-2004, caracterizado pela formação de um mercado financeiro internacional integrado; e iv) utiliza-se um amplo conjunto de índices de integração financeira internacional de jure e de facto e uma medida de fluxo líquido de capitais.

As evidências apresentadas ao longo do trabalho permitem dois conjuntos de conclusões: com base na metodologia econométrica adotada, não há evidências de que a integração financeira internacional e a importação líquida de capitais aumentam a taxa de convergência condicional nas economias nacionais; os capitais fluem em termos líquidos dos países em desenvolvimento para os países desenvolvidos e são alocados, entre os países em desenvolvimento, para aqueles com menor crescimento da produtividade total dos fatores. Essas evidências não corroboram as previsões do modelo neoclássico relativas ao padrão dos fluxos internacionais de capitais e às relações entre integração financeira internacional, fluxos internacionais de capitais e crescimento econômico e são, portanto, uma crítica contundente à tese de crescimento com poupança externa.

O modelo neoclássico prevê que, em um mundo onde a única distorção fosse a existência de controles sobre os fluxos internacionais de capitais, a remoção desses controles resultaria em ganhos, especialmente para os países em desenvolvimento. A assunção da existência de múltiplas distorções no mercado financeiro internacional e nas economias domésticas e os argumentos acerca das relações entre a integração financeira internacional, fluxos internacionais de capitais e apreciação da taxa real de câmbio explicitam as fragilidades dos fundamentos teóricos subjacentes a esse modelo. Dessa maneira, os resultados apresentados acerca da ausência de evidências a corroborarem as previsões do modelo neoclássico são coerentes com a discussão acerca das fragilidades dos fundamentos teóricos subjacentes a esse modelo.

\section{REFERÊNCIAS BIBLIOGRÁFICAS}

ACEMOGLU, D. (2009) Introduction to Modern Economic Growth. Princeton University Press.

AGHION, P.; HOWITT, P.; MAYER-FOULKES, D. (2005) "The effect of financial development on convergence: Theory and evidence", Quarterly Journal of Economics, 120 (1), p.173-222.

ARELLANO, M.; BOND, S. (1991) "Some tests of specification for panel data: Monte Carlo evidence and an application to employment equations", Review of Economic Studies, 58 (2), p.277-297.

ARELLANO, M.; BOVER, O. (1995) "Another look at the instrumental-variable estimation of error-components models", Journal of Econometrics, 68, p.29-51.

ARETA, C.; EICHENGREEN, B.; WYPLOSZ, C. (2001) "When does capital account liberalization help more than it hurts?”, National Bureau of Economic Research, Working Paper $n^{\circ} 8414$.

BARRO, R. J.; LEE, J. W. (2001) "International data on educational attainment: Update and implications", Oxford Economic Papers, 53 (3), p. 541-563.

BARRO, R.; SALA-I-MARTIN, X. (2004) Economic Growth. $2^{\text {a }}$ ed. Cambridge, Massachusetts: The MIT Press. 
BEKAERT, G.; HARVEY, C. R.; LUNDBLAD, C. (2005) “Does financial liberalization spur growth?”, Journal of Financial Economics, 77 (1), p.3-55.

BHAGWATI, J. (1998) The capital myth: The difference between trade in widgets and dollars", Foreign Affairs, 77 (3), p. 7-12.

BLUNDELL, R.; BOND, S. (1998) "Initial conditions and moment restrictions in dynamic panel data models", Journal of Econometrics, 87, p.115-143.

BRESSER-PEREIRA, L. C.; GALA, P. (2007) "Por que a poupança externa não promove crescimento", Revista de Economia Política, 27 (1), p.3-19.

CARKOVIC, M.; LEVINE, R. (2005) “Does foreign direct investment accelerate economic growth?”, In: MORAN, T. H.; GRAHAM, E. M.; BLOMSTROM, M. Does Foreign Direct Investment Promote Development? Washington: Institute for International Economics.

CHINN, M. D.; ITO, H. (2007) A New Measure of Financial Openness. Disponível em: <http://www. ssc.wisc.edu/ mchinn/kaopen_Chinn-Ito.pdf>. Acesso: Maio de 2007.

DAMASCENO, A. O. (2007) "Integração financeira internacional e crescimento econômico: Uma crítica à abordagem convencional”, Economia e Sociedade, 16 (2), p.171-198.

DAMASCENO, A. O. (2008) "Liberalização da conta de capitais e crescimento econômico: evidências de dados em painel para América Latina”, Revista de Economia Política, 28 (4), p.595-611.

DELL'ARICCIA, G.; DI GIOVANNI, J.; FARIA, A.; KOSE, M. A.; MAURO, P.; SCHINDLER, M.; TERRONES, M. OSTRY, J. D. (2008) "Reaping the benefits of financial globalization". International Monetary Fund, Occasional Paper $\mathrm{n}^{\circ} 264$.

EDISON, H. J.; KLEIN, M. W.; RICCI, A. L.; SLØK, T. (2004) “Capital account liberalization and economic performance: survey and synthesis”, IMF Staff Papers, 51 (2) p.220-256.

EDISON, H. J.; LEVINE, R.; RICCI, A. L.; SLØK, T. (2002) "International financial integration and economic growth", Journal of International Money and Finance, 21 (6), p.749-776.

EDWARDS, S. (2005) “Capital Controls, sudden stops and current account reversals". National Bureau of Economic Research, Working Paper $\mathrm{n}^{\circ} 11170$.

EDWARDS, S. (2007) "Capital controls, capital flows contractions, and macroeconomic vulnerability”, Journal of International Money and Finance, 26 (5), p.814-840.

EICHENGREEN, B.; MUSSA, M.; DELL'ARICCIA, G.; DETRAGIACHE, E.; MILESI-FERRETTI, G. M.; TWEEDIE, A. (1998) “Capital account liberalization: theoretical and practical aspects”, International Monetary Fund, Occasional Paper $\mathrm{n}^{\circ} 172$.

EICHENGREEN, B. (2007) The Cautious Case for Capital Flows. University of California.

FARIA, J. A.; PAULA, L. F; PIRES, M. C; MEYER, T. R. (2009) "Liberalização financeira, performance econômica e estabilidade macroeconômica no Brasil: uma avaliação do período recente.” Texto para Discussão N.1436. IPEA: Rio de Janeiro.

GOURINCHAS, P. O.; JEANNE, O. (2006) "The elusive gains from international financial integration”, Review of Economic Studies, 73 (3), p.715-741.

GOURINCHAS, P. O.; JEANNE, O. (2008) "Capital flows to developing countries: The allocation puzzle”, National Bureau of Economic Research, Working Paper n ${ }^{\circ} 13602$.

GRILLI, V.; MLESI-FERRETTI, J. M. (2995) "Economic effects and structural determinants of capital controls”, IMF Staff Papers, 42 (3), p.517-551.

HENRY, P. B. (2007) “Capital account liberalization: Theory, evidence, and speculation”, Journal of Economic Literature, 45 (4), p.887-935.

INTERNATIONAL MONETARY FUND. (2008) World Economic Outlook Database.

INTERNATIONAL MONETARY FUND. (2008) International Financial Statistics.

KLEIN, M. W. (2005) "Capital account liberalization, institutional quality and economic growth: Theory and evidence". National Bureau of Economic Research, Working Paper $\mathrm{n}^{\circ}$ 11112, 2005.

KOSE, M. A.; PRASAD, E.; ROGOFF, K.; WEI, S. (2009) "Financial globalization: A reappraisal”, IMF Staff Papers, 56 (1), p.8-62.

KRAAY, A. (1998) "In Search of the macroeconomic effects of capital account liberalization", World Bank.

LANE, P. R.; MILESI-FERRETTI, G. M. (2007) “The external wealth of nations mark II: Revised and 
extended estimates of foreign assets and liabilities, 1970-2004", Journal of International Economics, 73 (2), p.263-294.

LUCAS, R. (1990) "Why doesn't capital flow from rich to poor countries?” The American Economic Review, 80 (2), p. 92-96.

McKENZIE, D. J. (2001) “The impact of capital controls on growth convergence”, Journal of Economic Development, 26 (1), p.1-25.

OREIRO, J. L.; PAULA, L. F.; SILVA, G. J.; ONO, F. H. (2005) “Conversibilidade da conta de capital, taxa de juros e crescimento: uma avaliação empírica da proposta de plena-conversibilidade do Real”, Revista de Economia Contemporânea, 9 (2).

POLITICAL SERVICE RISK GROUP. (2008) International Country Risk Guide. PSRG: Nova York.

PRASAD, E.; ROGOFF, K.; WEI, S.; KOSE, M. A. (2003) "Effects of financial globalization on developing countries: Some empirical evidence”, International Monetary Fund, Occasional Paper ${ }^{\circ}$ 220.

PRASAD, E.; RAJAN, R.; SUBRAMANIAN, A. (2007) "Foreign capital and economic growth", Brookings Papers on Economic Activity, 38 (1), p. 153-230.

QUINN, D. P. (1997) “The correlates of change in international financial regulation”, American Political Science Review, 91 (3), p.531-551.

ROCHA, M; OREIRO, J. L. (2009) Capital Accumulation, External Endebtness and Macroeconomic Performance of Emerging Economies. Anais do XXXVII Encontro Nacional de Economia, Foz do Iguaçu.

RODRIK, D. (1998) “Who needs capital-account convertitility?” In: PETER, B. K. (ed.). Should the IMF Pursue Capital-Account Convertitility? Princeton University, Department of Economics, Essays in International Finance $\mathrm{n}^{\circ} 207$.

RODRIK, D.; SUBRAMANIAN, A. (2009) “Why did financial globalization disappoint?” IMF Staff Papers, 56 (1), p.112-138.

ROODMAN, D. (2009) "How to do xtabond2: An introduction to difference and system GMM in Stata". The Stata Journal, 9 (1), p.86-136.

STIGLITZ, J. (2000) "Capital market liberalization, economic growth, and instability", World Development 28 (6), p. 1075-1086.

WINDMEIJER, F. (2005) "A finite sample correction for the variance of linear efficient two-step GMM estimators”, Journal of Econometrics, 126 (1), p.25-51.

WORLD BANK. (2007) World Development Indicators. 


\section{APÊNDICE}

Tabela 1: Integração Financeira Internacional e Convergência Condicional - CIIFI

\begin{tabular}{|c|c|c|c|c|c|c|c|c|}
\hline & \multicolumn{4}{|c|}{ Amostra Total } & \multicolumn{4}{|c|}{ Países em Desenvolvimento } \\
\hline & (1) & $(2)$ & (3) & (4) & (5) & (6) & (7) & (8) \\
\hline & $\begin{array}{c}\text { Sys- } \\
\text {-GMM }\end{array}$ & $\begin{array}{c}\text { Dif- } \\
- \text { GMM }\end{array}$ & $\begin{array}{l}\text { Sys- } \\
\text {-GMM }\end{array}$ & $\begin{array}{c}\text { Dif- } \\
\text {-GMM }\end{array}$ & $\begin{array}{c}\text { Sys- } \\
\text {-GMM }\end{array}$ & $\begin{array}{c}\text { Dif- } \\
\text {-GMM }\end{array}$ & $\begin{array}{c}\text { Sys- } \\
\text {-GMM }\end{array}$ & $\begin{array}{c}\text { Dif- } \\
- \text { GMM }\end{array}$ \\
\hline \multirow[t]{2}{*}{ PIB Inicial } & $-0,0051$ & $-0,2495$ & 0,0144 & $-0,2784$ & $-0,0121$ & $-0,2163$ & 0,0152 & $-0,2885$ \\
\hline & $(0,0255)$ & $(0,1061)^{* *}$ & $(0,0242)$ & $(0,0898)^{* * *}$ & $(0,0242)$ & $(0,0895)^{* *}$ & $(0,0245)$ & $(0,0878)^{* * *}$ \\
\hline \multirow[t]{2}{*}{ PIB Inicial x CIIFI } & $-0,0001$ & $-0,0001$ & $-0,0001$ & $-0,0001$ & 0,0000 & $-0,0003$ & $-0,0001$ & $-0,0003$ \\
\hline & $(0,0002)$ & $(0,0002)$ & $(0,0001)$ & $(0,0002)$ & $(0,0003)$ & $(0,0004)$ & $(0,0003)$ & $(0,0003)$ \\
\hline \multirow[t]{2}{*}{ CIIFI } & $-0,0001$ & 0,0004 & $-0,0001$ & 0,0003 & $-0,0001$ & 0,0003 & $-0,0002$ & 0,0000 \\
\hline & $(0,0003)$ & $(0,0004)$ & $(0,0004)$ & $(0,0004)$ & $(0,0003)$ & $(0,0004)$ & $(0,0004)$ & $(0,0005)$ \\
\hline$A R(2)$ & 0,136 & 0,688 & 0,193 & 0,942 & 0,592 & 0,654 & 0,988 & 0,851 \\
\hline Hansen Test & 0,403 & 0,262 & 0,693 & 0,207 & 0,920 & 0,606 & 1,000 & 0,499 \\
\hline Diff. Hansen Test & 0,553 & & 0,724 & & 0,929 & & 1,000 & \\
\hline № Observações & 310 & 228 & 304 & 222 & 225 & 165 & 220 & 160 \\
\hline
\end{tabular}

Nota: A variável dependente é a taxa de crescimento do PIB per-capita real e a variável PIB Inicial está em logaritmo. Nas equações apresentadas nas colunas 1, 2, 5 e 6 as variáveis incluídas e não reportadas são: taxa de crescimento do PIB per-capita real defasada em um período, dummies temporais e as variáveis Capital Humano, Lei \& Ordem e Comércio expressas em logaritmo. Nas equações apresentadas nas colunas 3, 4, 7 e 8 as variáveis incluídas e não reportadas são: taxa de crescimento do PIB per-capita real defasada em um período, dummies temporais e as variáveis Capital Humano, Lei \& Ordem, Comércio, Inflação e Governo expressas em logaritmo. Ademais, as equações estimadas por System GMM incluem uma constante, não reportada na tabela. * ${ }^{* *}$, ***, significativos a $10 \%, 5 \%$ e $1 \%$, respectivamente. Todas as estimações são two-step, os erros-padrão estão em parêntese e são corrigidos utilizando o procedimento desenvolvido por Windmeijer (2005). São reportados os p-valores das estatísticas de teste AR(2), Hansen Test e Diff. Hansen Test.

Tabela 2: Integração Financeira Internacional e Convergência Condicional — SEIFI

\begin{tabular}{|c|c|c|c|c|c|c|c|c|}
\hline & \multicolumn{4}{|c|}{ Amostra Total } & \multicolumn{4}{|c|}{ Países em Desenvolvimento } \\
\hline & (1) & (2) & (3) & (4) & (5) & (6) & (7) & (8) \\
\hline & $\begin{array}{l}\text { Sys- } \\
\text {-GMM }\end{array}$ & $\begin{array}{c}\text { Dif- } \\
\text {-GMM }\end{array}$ & $\begin{array}{l}\text { Sys- } \\
\text {-GMM }\end{array}$ & $\begin{array}{c}\text { Dif- } \\
\text {-GMM }\end{array}$ & $\begin{array}{l}\text { Sys- } \\
\text {-GMM }\end{array}$ & $\begin{array}{c}\text { Dif- } \\
\text {-GMM }\end{array}$ & $\begin{array}{l}\text { Sys- } \\
\text {-GMM }\end{array}$ & $\begin{array}{c}\text { Dif- } \\
\text {-GMM }\end{array}$ \\
\hline \multirow[t]{2}{*}{ PIB Inicial } & $-0,0076$ & $-0,2959$ & 0,0077 & $-0,2641$ & $-0,0168$ & $-0,2589$ & 0,0115 & $-0,2346$ \\
\hline & $(0,0258)$ & $(0,0665)^{* * *}$ & $(0,0224)$ & $(0,0761)^{* * *}$ & $(0,0342)$ & $(0,0885) * * *$ & $(0,0309)$ & $(0,1061)^{* *}$ \\
\hline \multirow[t]{2}{*}{ PIB Inicial $\times$ SEIFI } & $-0,0001$ & 0,0002 & $-0,0001$ & 0,0001 & 0,0000 & 0,0000 & $-0,0001$ & $-0,0001$ \\
\hline & $(0,0002)$ & $(0,0002)$ & $(0,0002)$ & $(0,0003)$ & $(0,0004)$ & $(0,0006)$ & $(0,0003)$ & $(0,0005)$ \\
\hline \multirow[t]{2}{*}{ SEIFI } & $-0,0004$ & 0,0010 & $-0,0005$ & 0,0009 & $-0,0006$ & 0,0006 & $-0,0007$ & 0,0003 \\
\hline & $(0,0005)$ & $(0,0006)$ & $(0,0005)$ & $(0,0006)$ & $(0,0005)$ & $(0,0012)$ & $(0,0005)$ & $(0,0010)$ \\
\hline
\end{tabular}




\begin{tabular}{lrrrrrrrr}
\hline AR(2) & 0,084 & 0,643 & 0,140 & 0,876 & 0,767 & 0,960 & 0,792 & 0,878 \\
Hansen Test & 0,386 & 0,420 & 0,565 & 0,421 & 0,834 & 0,668 & 0,998 & 0,480 \\
Diff. Hansen Test & 0,192 & & 0,186 & & 0,711 & & 1,000 & \\
№ Observações & 312 & 230 & 306 & 224 & 225 & 165 & 220 & 160 \\
\hline
\end{tabular}

Nota: Ver Tabela 1.

Tabela 3: Integração Financeira Internacional e Convergência Condicional — LMFIFI

\begin{tabular}{|c|c|c|c|c|c|c|c|c|}
\hline & \multicolumn{4}{|c|}{ Amostra Total } & \multicolumn{4}{|c|}{ Países em Desenvolvimento } \\
\hline & (1) & $(2)$ & (3) & (4) & (5) & (6) & (7) & (8) \\
\hline & $\begin{array}{l}\text { Sys- } \\
\text {-GMM }\end{array}$ & $\begin{array}{c}\text { Dif- } \\
\text {-GMM }\end{array}$ & $\begin{array}{c}\text { Sys- } \\
\text {-GMM }\end{array}$ & Dif-GMM & $\begin{array}{l}\text { Sys- } \\
\text {-GMM }\end{array}$ & $\begin{array}{c}\text { Dif- } \\
\text {-GMM }\end{array}$ & $\begin{array}{l}\text { Sys- } \\
\text {-GMM }\end{array}$ & Dif-GMM \\
\hline \multirow[t]{2}{*}{ PIB Inicial } & $-0,0361$ & $-0,2563$ & $-0,0165$ & $-0,2484$ & $-0,0305$ & $-0,2833$ & $-0,0127$ & $-0,2079$ \\
\hline & $(0,0171) * *$ & $(0,0767) * * *$ & $(0,0207)$ & $(0,1080)^{* *}$ & $(0,0147)^{* *}$ & $(0,0691) * * *$ & $+(0,0318)$ & $(0,0994) * *$ \\
\hline \multirow[t]{2}{*}{ PIB Inicial x LMFIFI } & 0,0018 & 0,0018 & $-0,0026$ & $-0,0009$ & 0,0042 & 0,0056 & 0,0003 & $-0,0065$ \\
\hline & $(0,0037)$ & $(0,0049)$ & $(0,0042)$ & $(0,0064)$ & $(0,0042)$ & $(0,0079)$ & $(0,0054)$ & $(0,0075)$ \\
\hline \multirow[t]{2}{*}{ LMFIFI } & $-0,0039$ & $-0,0161$ & 0,0064 & $-0,0097$ & $-0,0084$ & $-0,0172$ & $-0,0012$ & $-0,0093$ \\
\hline & $(0,0098)$ & $(0,0145)$ & $(0,0118)$ & $(0,0204)$ & $(0,0098)$ & $(0,0173)$ & $(0,0126)$ & $(0,0198)$ \\
\hline$A R(2)$ & 0,057 & 0,959 & 0,144 & 0,947 & 0,628 & 0,915 & 0,831 & 0,892 \\
\hline Hansen Test & 0,483 & 0,469 & 0,521 & 0,433 & 0,918 & 0,310 & 0,999 & 0,768 \\
\hline Diff. Hansen Test & 0,249 & & 0,231 & & 0,997 & & 1,000 & \\
\hline № Observações & 312 & 230 & 306 & 224 & 225 & 165 & 220 & 160 \\
\hline
\end{tabular}

Nota: Ver Tabela 1.

Tabela 4: Integração Financeira Internacional e Convergência Condicional — WDIIFI

\begin{tabular}{|c|c|c|c|c|c|c|c|c|}
\hline & \multicolumn{4}{|c|}{ Amostra Total } & \multicolumn{4}{|c|}{ Países em Desenvolvimento } \\
\hline & (1) & $(2)$ & (3) & (4) & (5) & (6) & (7) & (8) \\
\hline & Sys-GMM & $\begin{array}{c}\text { Dif- } \\
\text {-GMM }\end{array}$ & $\begin{array}{l}\text { Sys- } \\
\text {-GMM }\end{array}$ & $\begin{array}{r}\text { Dif- } \\
\text {-GMM }\end{array}$ & Sys-GMM & $\begin{array}{c}\text { Dif- } \\
\text {-GMM }\end{array}$ & $\begin{array}{l}\text { Sys- } \\
\text {-GMM }\end{array}$ & $\begin{array}{c}\text { Dif- } \\
- \text { GMM }\end{array}$ \\
\hline \multirow[t]{2}{*}{ PIB Inicial } & $-0,0293$ & 0,3191 & $-0,0112$ & $-0,2542$ & $-0,0230$ & $-0,2869$ & $-0,0161$ & $-0,2018$ \\
\hline & $(0,0128)^{* *}$ & $*(0,0803)^{* * *}$ & ${ }^{*}(0,0187)$ & $(0,0879)^{* * *}$ & $(0,0164)$ & $(0,0737)^{* * *}$ & $(0,0260)$ & $(0,1064)^{*}$ \\
\hline \multirow[t]{2}{*}{ PIB Inicial x WDIIFI } & 0,0001 & 0,0004 & $-0,0000$ & 0,0003 & 0,0004 & 0,0012 & 0,0004 & 0,0010 \\
\hline & $(0,0002)$ & $(0,0002)$ & $(0,0002)$ & $(0,0002)$ & $(0,0001)^{* *}$ & $(0,0002)^{* * *}$ & $(0,0002) *$ & $(0,0002)^{* * *}$ \\
\hline \multirow[t]{2}{*}{ WDIIFI } & $-0,0004$ & $-0,0014$ & $-0,0001$ & $-0,0012$ & $-0,0008$ & $-0,0024$ & $-0,0010$ & $-0,0022$ \\
\hline & $(0,0006)$ & $(0,0005)^{* * *}$ & $*(0,0006)$ & $(0,0005)^{* *}$ & $(0,0004) *$ & $(0,0004) * * *$ & $(0,0006)^{*}$ & $(0,0004)^{* * *}$ \\
\hline
\end{tabular}




\begin{tabular}{lrrrrrrrr}
\hline AR(2) & 0,069 & 0,721 & 0,169 & 0,739 & 0,601 & 0,683 & 0,708 & 0,696 \\
Hansen Test & 0,462 & 0,508 & 0,775 & 0,455 & 0,977 & 0,546 & 0,995 & 0,820 \\
Diff. Hansen Test & 0,462 & & 0,850 & & 0,988 & 0,697 & 1,000 & \\
№ Observações & 303 & 220 & 297 & 215 & 216 & 155 & 211 & 151 \\
\hline
\end{tabular}

Nota: Ver Tabela 1.

Tabela 5: Integração Financeira Internacional e Convergência Condicional — FLCTC

\begin{tabular}{|c|c|c|c|c|c|c|c|c|}
\hline & \multicolumn{4}{|c|}{ Amostra Total } & \multicolumn{4}{|c|}{ Países em Desenvolvimento } \\
\hline & (1) & $(2)$ & (3) & (4) & (5) & (6) & (7) & (8) \\
\hline & Sys-GMM & $\begin{array}{c}\text { Dif- } \\
\text {-GMM }\end{array}$ & $\begin{array}{c}\text { Sys- } \\
\text {-GMM }\end{array}$ & $\begin{array}{c}\text { Dif- } \\
\text {-GMM }\end{array}$ & $\begin{array}{c}\text { Sys- } \\
\text {-GMM }\end{array}$ & $\begin{array}{c}\text { Dif- } \\
\text {-GMM }\end{array}$ & $\begin{array}{c}\text { Sys- } \\
\text {-GMM }\end{array}$ & Dif-GMM \\
\hline \multirow[t]{2}{*}{ PIB Inicial } & $-0,0379$ & $-0,3187$ & $-0,0239$ & $-0,2773$ & $-0,0261$ & $-0,2756$ & $-0,0230$ & $-0,2588$ \\
\hline & $(0,0117)^{* * *}$ & $0,0681)^{* * *}$ & $(0,0145)^{*}$ & $, 0718)^{* * *}$ & $(0,0170)$ & $(0,0679)^{* * *}$ & $(0,0216)$ & $(0,0876)^{* * *}$ \\
\hline \multirow[t]{2}{*}{ PIB Inicial x FLCTC } & 0,0009 & $-0,0023$ & 0,0006 & $-0,0015$ & 0,0014 & 0,0000 & 0,0013 & 0,0008 \\
\hline & $(0,0006)$ & $(0,0016)$ & $(0,0011)$ & $(0,0015)$ & $(0,0012)$ & $(0,0022)$ & $(0,0009)$ & $(0,0022)$ \\
\hline \multirow[t]{2}{*}{ FLCTC } & 0,0019 & 0,0019 & 0,0014 & 0,0015 & 0,0007 & 0,0004 & 0,0013 & 0,0014 \\
\hline & $(0,0014)$ & $(0,0023)$ & $(0,0016)$ & $(0,0027)$ & $(0,0020)$ & $(0,0021)$ & $(0,0017)$ & $(0,0019)$ \\
\hline$A R(2)$ & 0,120 & 0,654 & 0,243 & 0,761 & 0,722 & 0,799 & 0,911 & 0,766 \\
\hline Hansen Test & 0,551 & 0,270 & 0,570 & 0,374 & 0,883 & 0,251 & 0,998 & 0,445 \\
\hline Diff. Hansen Test & 0,555 & & 0,834 & & 1,000 & & 1,000 & \\
\hline № Observações & 312 & 230 & 306 & 224 & 225 & 165 & 220 & 160 \\
\hline
\end{tabular}

Nota: Ver Tabela 1. 